\title{
Comparison of Wake-Vortex Parameters Measured by Pulsed and Continuous-Wave Lidars
}

\author{
Friedrich Köpp, ${ }^{*}$ Stephan Rahm, ${ }^{\dagger}$ and Igor Smalikho ${ }^{\ddagger}$ \\ DLR, German Aerospace Research Center, D-82234 Wessling, Germany \\ Agnès Dolfi ${ }^{\S}$ and Jean-Pierre Cariou ${ }^{\text {II }}$ \\ ONERA, 91761 Palaiseau, France \\ and \\ Michael Harris** and Robert I. Young ${ }^{\dagger \dagger}$ \\ QinetiQ Malvern, Worcestershire, England WR14 3PS, United Kingdom
}

\begin{abstract}
Field trials carried out at Tarbes airfield in the summer of 2002 offered the unique opportunity to compare the results of simultaneous wake-vortex measurements by the $2-\mu \mathrm{m}$ pulsed Doppler lidar from DLR, German Aerospace Research Center, and the 10- $\mu \mathrm{m}$ continuous wave (cw) Doppler lidars from ONERA and QinetiQ. The discrepancies in vortex core position obtained from the data of the pulsed lidar and the cw lidars are $9 \mathrm{~m}$ for the vertical and $13 \mathrm{~m}$ for the horizontal co-ordinates. The accuracies of the vortex circulation measurements with the DLR and ONERA lidars are almost the same and equal $13 \mathrm{~m}^{2} / \mathrm{s}$. This accuracy and the long-range capability of the pulsed lidar allows precise measurements over long periods from the moment of wake generation to a progressed state of vortex decay. Moreover, the influence of different atmospheric turbulence conditions and aircraft configurations on the wake-vortex circulation can be analyzed. This has been demonstrated out of ground effect under conditions of weak to moderate levels of turbulence.
\end{abstract}

\section{Introduction}

$\mathbf{T}$ HE wake vortices generated by aircraft can be dangerous to other aircraft following closely behind. The duration and trajectories of the vortices are strongly dependent on the atmospheric conditions (wind and turbulence) in which they form. The International Civil Aviation Organization has defined minimum separation distances, which are the main factors for capacity limitations at larger airports. A better understanding of the evolution and behavior of wake vortices in their atmospheric environment could lead to a relaxation of the separation distances and to an enhancement of airport throughput.

Coherent Doppler lidar (CDL) is a powerful technique both for the measurement of wind and atmospheric turbulence ${ }^{1-10}$ and for the detection and study of aircraft wake vortices. ${ }^{1-21}$ There are two types of CDL: continuous wave (cw) and pulsed. Both types have been successfully used for measurements of the wake-vortex characteristics. ${ }^{19,21}$ To minimize the uncertainty in the distance between the cw lidar and the vortex core, the measurements can be simultaneously carried out with two cw lidars, and the vortex trajectories can be derived using the triangulation method. This strategy was successfully tested in spring 2001 in a field trial at the

Received 9 February 2004; revision received 13 July 2004; accepted for publication 20 July 2004. Copyright (C) 2004 by the American Institute of Aeronautics and Astronautics, Inc. All rights reserved. Copies of this paper may be made for personal or internal use, on condition that the copier pay the $\$ 10.00$ per-copy fee to the Copyright Clearance Center, Inc., 222 Rosewood Drive, Danvers, MA 01923; include the code 0021-8669/05 \$10.00 in correspondence with the CCC.

*Research Scientist, Lidar Group, Institute of Atmospheric Physics, Oberpfaffenhofen.

${ }^{\dagger}$ Research Scientist, Lidar Group, Institute of Atmospheric Physics, Oberpfaffenhofen.

${ }^{\ddagger}$ Research Scientist, Lidar Group, Institute of Atmospheric Physics, Oberpfaffenhofen.

${ }^{\S}$ Research Scientist, Départment Optique Théorique et Appliquée, Chemin de la Hunière.

IIManager, Laser Applications and Optoelectronics Unit, Départment Optique Théorique et Appliquée, Chemin de la Hunière.

** QinetiQ Fellow, Sensor and Electronics Division.

${ }^{\dagger}$ Project Manager, Sensor and Electronics Division.
Fairchild-Dornier airfield, Oberpfaffenhofen, Germany, under the European Commission (EC) Project C-Wake. ${ }^{19}$ An integration method ${ }^{20}$ can be applied for estimation of the vortex circulation from the measured dependence of the tangential velocity on the distance from the vortex center. A similar approach of circulation estimation from data measured with a pulsed lidar was presented in Ref. 21. However, in contrast to the $\mathrm{cw}$ lidar, the distance between the pulsed lidar and the vortex core can be determined with acceptable accuracy (relative error $\sim 1 \%$ ) from data measured with one pulsed lidar only.

In summer 2002, three lidar teams participated in the field experiment at Tarbes airfield, France, in the frame of the EC Project C-Wake. Two cw 10- $\mu \mathrm{m}$ lidars from ONERA, France, and QinetiQ, United Kingdom and one pulsed $2-\mu \mathrm{m}$ lidar from DLR, German Aerospace Research Center, Germany, were deployed. In this paper, we present the results of the lidar measurements of the wake vortices generated by a large transport aircraft (LTA). The trajectories and circulation of the vortices measured with the pulsed lidar and the two cw lidars (on the basis of the triangulation results obtained from data measured with the ONERA and QinetiQ lidars) are compared. The behavior of the vortex circulation vs time at various atmospheric turbulence conditions will be shown.

\section{CDL}

The principle of CDL is based on the emission of laser radiation into the atmosphere and the coherent detection of the light backscattered by aerosol particles. The information about the velocity (projection of the velocity vector on the probing laser beam axis or radial velocity) of the particles moving with the airflow is extracted from the measured Doppler frequency shift of the backscattered wave. The main elements of a coherent Doppler lidar are laser, telescope, local oscillator, and detector. For variation of the angle of the probing laser beam direction, a scanning device is used.

\section{2-Micrometer Pulsed Lidar}

The $2-\mu \mathrm{m}$ pulsed Doppler lidar deployed by DLR at Tarbes airfield for wake-vortex measurements is based on the transceiver unit MAG-1 from CLR Photonics. ${ }^{22}$ It comprises the cw master laser for injection seeding of the slave laser and for acting as local oscillator 
for coherent detection and the slave laser for transmission of the pulses of 2-mJ energy and 400-ns length into the atmosphere with a pulse repetition rate of $500 \mathrm{~Hz}$. Both lasers are diode-pumped Tm:LuAG lasers with optimum matching to the atmospheric window at 2022-nm wavelength. The offset frequency of $100 \pm 3 \mathrm{MHz}$ between both lasers is generated by an acousto-optic modulator. The diameter of the transmit-receive telescope is $108 \mathrm{~mm}$. The analog signal from the signal detector is amplified by a $1-\mathrm{GHz}$ amplifier with adjustable gain of 30-70 dB. This amplified signal and the reference signal from the pulse-monitor detector are fed to the data acquisition and recording unit. These data are digitized with a sampling rate of $500 \mathrm{MHz}$. The measurement plane is linearly scanned by an oscillating mirror of $200 \mathrm{~mm}$ diameter with a scan speed of $2 \mathrm{deg} / \mathrm{s}$ and a flyback time of $0.5 \mathrm{~s}$. A detailed description of the $2-\mu \mathrm{m}$ lidar is given in Ref. 17.

For post-trial processing of the data measured in Tarbes, always 256 samples of the backscatter signal from each single shot at any chosen range were used for the analysis of the radial velocities in the sensing volume. In this case, the effective longitudinal size of the sensing volume equals $88 \mathrm{~m}$ (Refs. 8 and 21), and it does not depend on the range (distance between the lidar and the sensing volume center).

\section{0-Micrometer CW Lidars}

During the Tarbes experiment, the lidar teams of ONERA and QinetiQ were operating similar systems based on a $\mathrm{CO}_{2}$ laser source emitting several watts of eye-safe radiation. Details of the lidar systems may be obtained from Refs. 13 and 17. The lidars are monostatic, using common transmit and receive optics with a $30-\mathrm{cm}$ telescope aperture. The probing laser beam can be focused to give a range resolution $\Delta R$ (effective longitudinal size of the sensing volume), which depends on the focal length $R$ as $\Delta R \sim R^{2}$ (Ref. 23). At $R=100 \mathrm{~m}, \Delta R \approx 10 \mathrm{~m}$, and at $R=300 \mathrm{~m}$, the sizes of the sensing volume in the cases of $\mathrm{cw}$ and pulsed lidars are approximately the same, that is, $\Delta R \approx 88 \mathrm{~m}$. The real-time signal processing is based on either surface acoustic wave or digital spectral analysis. A single spectrum is obtained within $50 \mu \mathrm{s}$. However, integration of several spectral estimates must be performed to achieve an adequate signal-to-noise ratio (SNR). Averaged velocity spectra are acquired at rates of 33 (QinetiQ lidar) and 100 (ONERA lidar) per second with a resolution of approximately $0.1 \mathrm{~m} / \mathrm{s}$. The probing laser beams were scanned with a speed of $10 \mathrm{deg} / \mathrm{s}$ (QinetiQ lidar) and $12 \mathrm{deg} / \mathrm{s}$ (ONERA lidar).

\section{Experiment at Tarbes Airfield}

As part of the EC Project C-Wake, an extended field campaign was carried out in June 2002 with pertinent observations made during four days, 12 June 2002-14 June 2002 and 17 June 2002, at the airfield of Tarbes, France. There, a large number of different sensors $^{24}$ and three coherent Doppler lidars (the $2-\mu \mathrm{m}$ pulsed lidar from DLR, Germany, and two $10-\mu \mathrm{m}$ cw lidars from QinetiQ, United Kingdom and from ONERA, France) were deployed for simultaneous investigation of the wake vortices and for observation of the atmospheric environment.

Figure 1 shows the geometry of the wake-vortex measurements with three Doppler lidars. The $10-\mu \mathrm{m}$ cw lidars from QinetiQ (L2) and from ONERA (L3) were positioned at a distance of 702 and $824 \mathrm{~m}$, respectively, from the $2-\mu \mathrm{m}$ pulsed lidar for precise measurement of velocity profiles in the late phase of the vortex life cycle. The distance between the cw lidars (L2 and L3) is $122 \mathrm{~m}$. During the measurements, lidars L2 and L3 were scanning in the vertical plane $(Z, Y)$. The scan sector angle was, on average, $60 \mathrm{deg}$ for both lidars (elevation angles 40-100 deg for lidar L2 and $80-140$ or $70-130$ deg for lidar L3). The pulsed lidar L1 was scanning in a slightly different vertical plane, where the angle between this plane and $(Z, Y)$ plane was approximately $7.7 \mathrm{deg}$. In the case of lidar L1, the scan angles were 0-20 deg (12 June 2002), 3-25 deg (13 June 2002), 2-24 deg (14 June 2002), and 0-21 deg (17 June 2002).
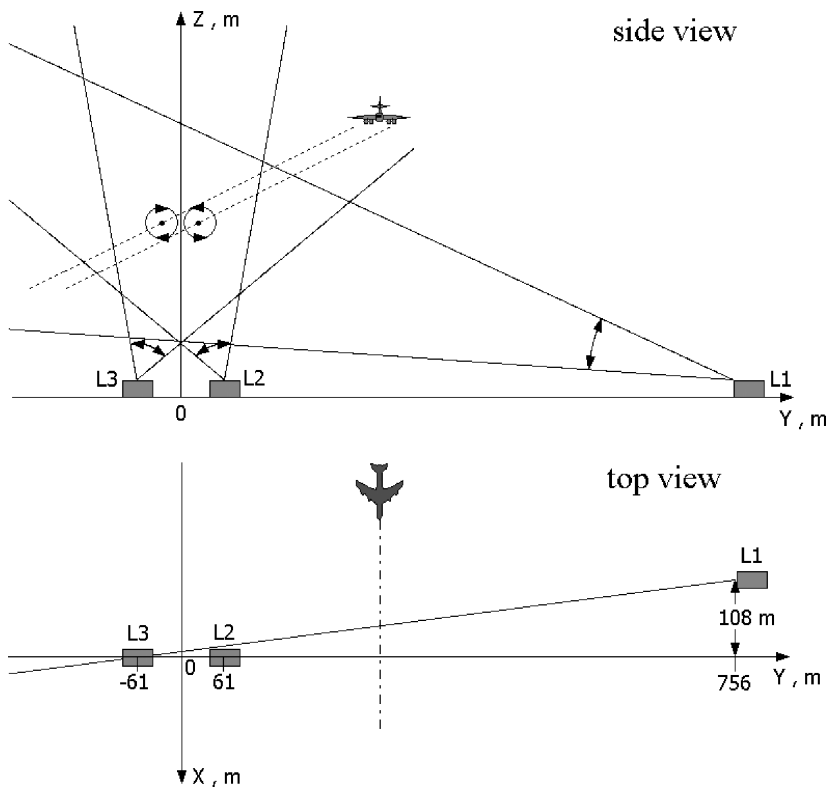

Fig. 1 Geometry of lidar measurements at Tarbes airfield; L1, 2- $\mu \mathrm{m}$ pulsed lidar and $\mathrm{L} 2$ and $\mathrm{L3}, 10-\mu \mathrm{m}$ cw lidars.

During the four days of the experiment, a LTA-type test aircraft was available performing 65 overflights. The aircraft flew along the line parallel to the $X$ axis (dashed-dotted line in Fig. 1) at different heights $Z(180-430 \mathrm{~m})$ and distances $Y$ between the vertical flight plane and $(Z X)$ plane. (Maximal $Y \approx 500 \mathrm{~m}$.)

The crosswind (the projection of the wind vector on the $Y$ axis) has an essential influence on the wake-vortex trajectories. In strong crosswind conditions, for example, the vortices remain only a short period within the measurement area. (See overlapping scan sectors of lidars L2 and L3 in Fig.1.) On the first day of the measurements, the crosswind was positive. (The crosswind direction is from the side of lidar L1 to lidars L2/L3.) It was negative during the other three days. Because we had strong crosswind (up to $6 \mathrm{~m} / \mathrm{s}$ ) on the first day (12 June 2002), only few overflights were performed on this day. On the other days, the crosswind was $4-4.5 \mathrm{~m} / \mathrm{s}$ (13 June 2002) and 2-2.5 m/s (14 June 2002 and 17 June 2002). With the exception of the first day, the measurements were carried out in the evening hours (18:00-22:00 local time). The weather conditions were favorable during the measurements: The sky was cloudless, and a lot of aerosol particles were present in the atmosphere providing relatively high SNRs.

\section{Data Processing}

After the experimental campaign, the raw data (the signals measured by the pulsed lidar and the spectra measured by the $\mathrm{cw}$ lidars) were processed to estimate the wake-vortex characteristics such as core position (coordinate) and circulation.

\section{Processing of Data Measured by Pulsed Lidar}

The processing of the data measured by the $2-\mu \mathrm{m}$ pulsed Doppler lidar includes four main stages, the estimation of 1) the Doppler spectra (spectra of the power of coherently detected backscatter signals), 2) the radial velocity and velocity envelopes, 3) the vortex core position, and 4 ) the vortex circulation. The detailed description of the processing procedure is given in Ref. 21.

To estimate single Doppler spectra, the measured data of 25 shots were used for spectral accumulation. Taking into account that the scan speed is $2 \mathrm{deg} / \mathrm{s}$ and the pulse repetition frequency is $500 \mathrm{~Hz}$, the elevation angle resolution is $0.1 \mathrm{deg}$, and at range of $R=600 \mathrm{~m}$, the vertical resolution is approximately $1 \mathrm{~m}$. These accumulated spectra were estimated every $3 \mathrm{~m}$ along the beam axis from $R=500 \mathrm{~m}$ up to $R=1100 \mathrm{~m}$. The scattering particles in the sensing volume (with a longitudinal size of $88 \mathrm{~m}$ ) have some velocity distribution. From the measured Doppler spectrum, one can estimate the mean radial velocity and, also, positive and negative velocity 
envelopes (or maximal and minimal radial velocity inside the sensing volume) using an appropriate threshold for the spectrum. ${ }^{21}$ From the obtained two-dimensional distributions of the radial velocity $V_{r}$ and the velocity envelopes $V_{ \pm}$vs range $R$ and elevation angle $\varphi$, the core positions $\left(R_{C l}\right.$ and $\left.\varphi_{C l}\right)$ of the right $(l=1)$ and left $(l=2)$ vortex can be found. Moreover, the velocity envelopes can be corrected by subtracting the background radial wind velocity. The vortex circulation $\Gamma$ is estimated from the corrected velocity envelopes $V_{ \pm}\left(R_{C 1}, \varphi\right)$ and $V_{ \pm}\left(R_{C 2}, \varphi\right)$ and the positions $R_{C l}$ and $\varphi_{C l}$ by formulas (2) and (3) given in Ref. 21.

\section{Processing of Data Measured by CW Lidars}

From the Doppler spectra measured by the cw lidars L2 and L3, the velocity envelopes $V_{ \pm}^{L 2}[\varphi(t)]$ and $V_{ \pm}^{L 3}[\varphi(t)]$ as functions of time $t$ are derived. For each overflight, the lidars acquire data containing several intersections with the vortex cores. These intersections provide the angular information used subsequently to derive vortex location by the triangulation methodology. ${ }^{19}$ The two series of angular measurements from each lidar can then be combined to estimate the core coordinates, as well as the ranges, that is, the distances between the lidars and the vortex cores. The elevation angles of the core intersection $\varphi_{C l}^{L 2}(t)$ and $\varphi_{C l}^{L 3}\left(t^{\prime}\right)$ (where as before $l=1$ for right and $l=2$ for left vortex) are extracted from the envelopes $V_{ \pm}^{L 2}$ and $V_{ \pm}^{L 3}$, where, as a rule, $t \neq t^{\prime}$. Because the distance between the lidars is known, the vortex core position can be found by application of the triangulation method. The direct interpolation (for example, linear interpolation of angles measured in adjoining time moments) of $\varphi_{C l}^{L 2}(t)$ or $\varphi_{C l}^{L 3}\left(t^{\prime}\right)$ to obtain the angles at the same time $t=t^{\prime}$ can not often give triangulation result with acceptable accuracy because of random variations of the estimated angles. For the triangulation process, we used the model extended kalman filter (EKF), ${ }^{25}$ in which the motions of the two vortex cores are considered to be linked to some degree. Detailed description of the processing procedure with EKF intended for the estimation of the vortex core coordinates $Z_{C l}$, $Y_{C l}$, and ranges $R_{C l}^{L 2}$ and $R_{C l}^{L 3}$, that is, distances between $l$ th vortex core and L2 and L3 lidar, respectively, is given in Ref. 19. For estimation of the vortex circulation $\Gamma$, the integration method ${ }^{20}$ is used, where the input parameters are $V_{ \pm}^{L m}(\varphi), \varphi_{C l}^{L m}$ and $R_{C l}^{L m}$, where $m=2$ and 3 is the lidar label.

Before the calculation of the vortex circulation from the data measured both by the pulsed lidar and the cw lidars, it is necessary to choose in the velocity envelopes $V_{ \pm}^{L m}(\varphi)$ subarrays at the regions around $\varphi_{C l}^{L m}$, where the tangential velocity of one separate vortex approaches a potential dependence on the distance $r=R_{C l}^{L m}\left|\sin \left(\varphi-\varphi_{C l}^{L m}\right)\right|$, that is, the tangential velocity equals $\Gamma /(2 \pi r), m=1,2,3$, and $l=1,2$. In the case of LTA-type aircraft, a minimum distance from the vortex core of $B / 12$ and a maximum distance of $B / 4$ (where $B$ is wingspan) are optimal for the integration boundaries. Figure 2 shows the scheme of data processing and results comparison.

The quality of the data measured with a cw Doppler lidar depends on the difference between the distance from the lidar to the vortex core and the focal length $R$. The larger such difference, the worse is the quality of the measured data. During the measurements with the QinetiQ lidar, the focal length $R$ was mostly fixed at a constant distance in the range of $120-170 \mathrm{~m}$. The wake vortices descend and move with the wind, and therefore, the distance from the lidar to the vortex core is changed during the measurement. As a result, for many cases the accuracy of the velocity envelope measured with the QinetiQ lidar is poor for the circulation estimation, but it is quite sufficient for the estimation of the core intersections $\varphi_{C l}^{L 2}$ that can then be used for triangulation. To improve the quality of the data, the focal length $R$ was changed in accordance with the result of simulating the wake vortex trajectory for each overflight. This has been applied for the measurements with the ONERA lidar. For example, during time the interval of $4 \mathrm{~min}, R$ can be changed from 300 to $110 \mathrm{~m}$. The input parameters for the simulation program are the aircraft height and the crosswind velocity. This program also takes into account the vortex descent. The quality of the data measured with the ONERA lidar is high almost for all overflights.

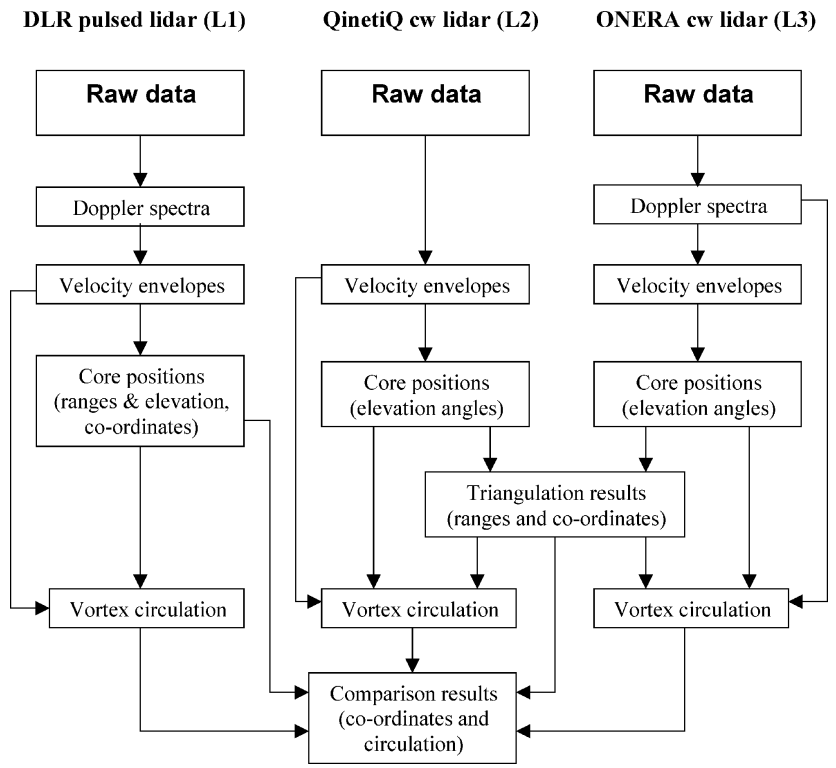

Fig. 2 Sequence of data processing and comparison of results.

\section{Comparison of Results of Wake-Vortex Measurements}

After an aircraft overflight, the generated vortex pair moves with the wind and simultaneously descends (if no strong upward atmospheric airflow occurs). Because of the different measurement geometry (Fig. 1) of the pulsed and cw lidars, the duration of the vortex measurements with lidar L1, respectively, lidars L2 and L3 are different. Figure 1 (side view) shows the scan sectors. It is obvious that the pulsed lidar L1 can measure older vortices compared with the cw lidars L2 and L3. The final results of measurement and data processing are the coordinates of the vortex cores and the vortex circulation vs time after overflight. The sampling of the wake-vortex trajectories and circulation by the pulsed and cw lidars occur at different moments of time. To compare the measured characteristics, it is necessary to use interpolation or temporal averaging over chosen intervals.

\section{Comparison of Vortex-Core Trajectories}

Figure 3 shows two examples of wake-vortex trajectories measured with the pulsed lidar (dashed curves) and with the cw lidars, that is, the triangulation result (solid curves). The measurements were carried out on 13 June 2002 and 14 June 2002 at crosswind velocities of $4.5 \mathrm{~m} / \mathrm{s}$ (Fig. 4a) and $2.5 \mathrm{~m} / \mathrm{s}$ (Fig. 4b), respectively. The crosswind was extracted from the data measured with the pulsed lidar (radial velocity estimates). The sequences of the circles (for the right vortex) and squares (for the left vortex) on the curves are the vortex core coordinates measured at the same time every $11 \mathrm{~s}$ both with the pulsed lidar and with the cw lidars. To obtain the simultaneous estimates of the vortex core coordinates, a linear interpolation between adjacent points was used. For the examples shown in Fig. 3, the aircraft coordinates as it passed through the $\mathrm{cw}$ lidar scan plane $(Y Z$ plane at $X=0)$ are $Z_{a}=334 \mathrm{~m}, Y_{a}=424 \mathrm{~m}$ (Fig. 4a) and $Z_{a}=347 \mathrm{~m}, Y_{a}=297 \mathrm{~m}$ (Fig. 4b). The vortex trajectories were measured with the pulsed lidar in the time intervals of 52-138 s (Fig. 4a) and 70-224 s (Fig. 4b) after overflight. In the case of the triangulation results (measurements with the $\mathrm{cw}$ lidars) the corresponding time intervals are 39-96 s (Fig. 4a) and 57-182 s (Fig. 4b). In these two examples, the time of the wake-vortex observation by the pulsed lidar is approximately 1 min longer than the observation time of the cw lidars. This is mainly due to the longer measurement range of the pulsed lidar L1 and the different geometry of the lidar measurements (Fig. 1).

After the interpolation to obtain simultaneous estimates of the core coordinates, a comparison was made of the positions of the vortex cores measured by the pulsed lidar and the cw lidars. In Fig. 4, the vortex core heights $Z$ derived from the cw lidar measurements are plotted against the corresponding heights for pulsed lidar. 

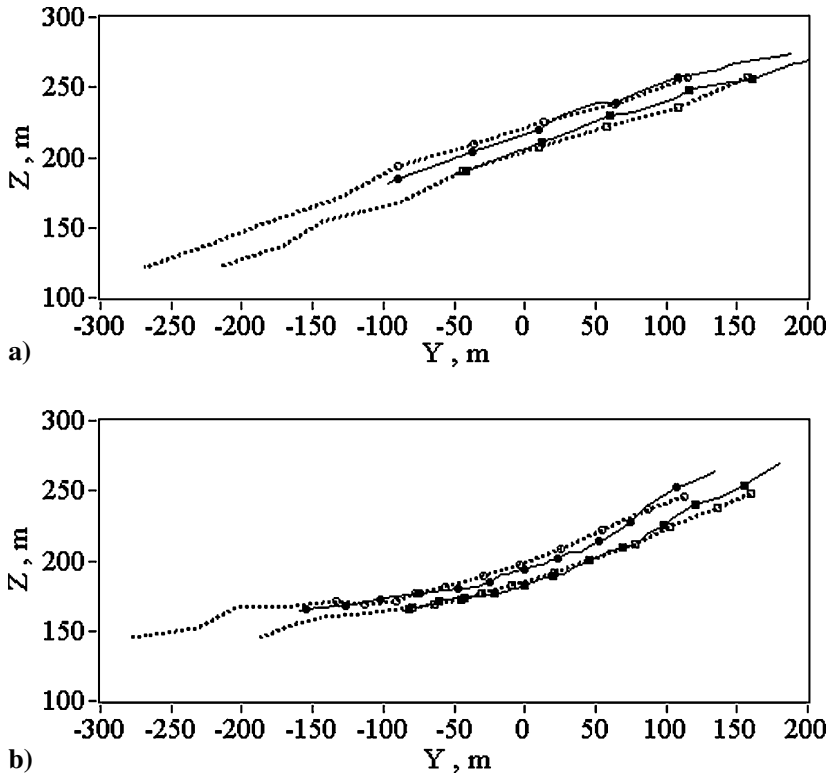

Fig. 3 Vortex core coordinates: ---, data measured with pulsed lidar and; - , triangulation for; $\bigcirc$, right vortex; and $a$, left vortex measured 11 s each with pulsed and cw lidars simultaneously: a) 13 June 2002 and b) 14 June 2002.

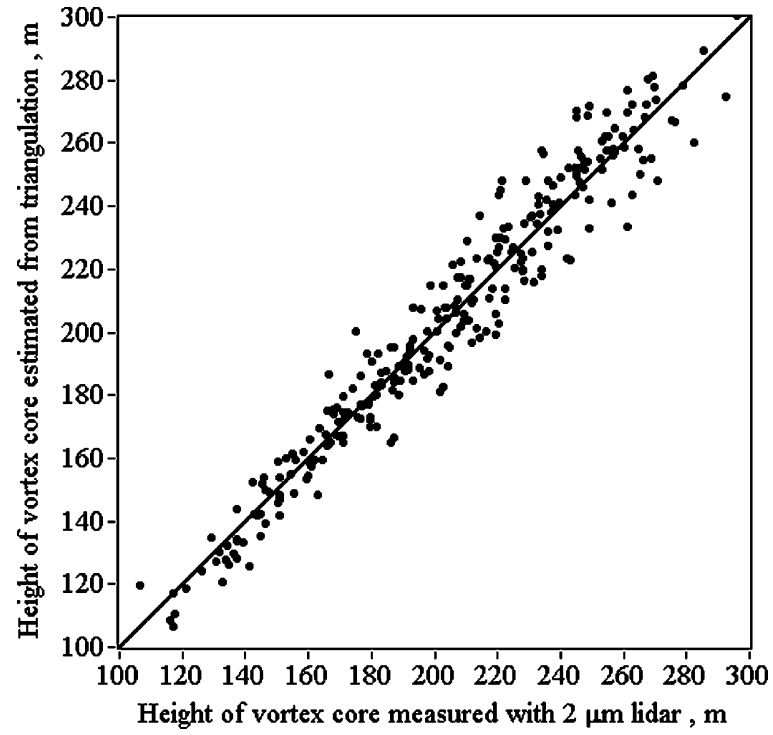

Fig. 4 Vortex core heights measured simultaneously with pulsed and cw lidars.

Similarly, Fig. 5 shows a comparison of the horizontal coordinate $Y$. In both Figs. 4 and 5, the points are concentrated around the line corresponding to the ideal case of perfect coincidence of the results. It can be seen that the scatter of the points in Fig. 4 becomes larger with increasing height of the core position.

For comparison of the measured results, two statistical parameters are introduced, $\hat{\sigma}_{Z}$ and $\hat{\sigma}_{Y}$, which characterize the level of discrepancy in the core coordinates obtained from the data of the pulsed lidar and the cw lidars, as

$$
\begin{gathered}
\hat{\sigma}_{Z}=\sqrt{\frac{1}{K} \sum_{k=1}^{K}\left[Z^{L 1}\left(t_{k}\right)-Z^{L 2,3}\left(t_{k}\right)\right]^{2}} \\
\hat{\sigma}_{Y}=\sqrt{\frac{1}{K} \sum_{k=1}^{K}\left[Y^{L 1}\left(t_{k}\right)-Y^{L 2,3}\left(t_{k}\right)\right]^{2}}
\end{gathered}
$$

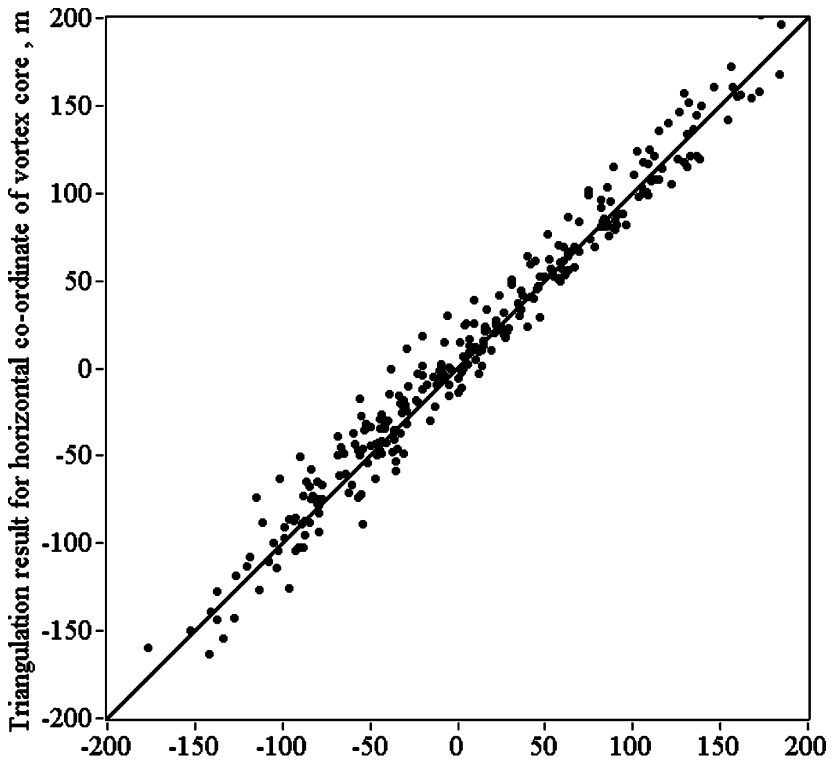

Horizontal co-ordinate of vortex core measured with $2 \mu \mathrm{m}$ lidar, $\mathrm{m}$

Fig. 5 Vortex core horizontal coordinates measured simultaneously with pulsed and cw lidars.

where $\left\{Z^{L 1}\left(t_{k}\right), Y^{L 1}\left(t_{k}\right)\right\}$ and $\left\{Z^{L 2,3}\left(t_{k}\right), Y^{L 2,3}\left(t_{k}\right)\right\}$ are the core coordinates measured by the pulsed lidar L1 and the cw lidars L2 and L3, whose measured data were used for the process of triangulation, respectively, at the time moment $t_{k}$, and $K$ is the total number of estimates used for averaging. In accordance with the data of Figs. 4 and $5, K=284$, and the calculations by Eqs. (1) and (2), we obtain the results $\hat{\sigma}_{Z} \approx 9 \mathrm{~m}$ and $\hat{\sigma}_{Y} \approx 13 \mathrm{~m}$. For $K=284$, the relative standard deviation of the estimates $\hat{\sigma}_{Z}$ and $\hat{\sigma}_{Y}$ is not larger than $10 \%$.

We can assume that the random errors of the estimates $\left\{Z^{L 1}\left(t_{k}\right), Y^{L 1}\left(t_{k}\right)\right\}$ and $\left\{Z^{L 2,3}\left(t_{k}\right), Y^{L 2,3}\left(t_{k}\right)\right\}$ are independent, statistically stationary, and homogeneous. Then, the variance $\sigma_{Z}^{2}=$ $\left\langle\hat{\sigma}_{Z}^{2}\right\rangle \quad\left(\sigma_{Y}^{2}=\left\langle\hat{\sigma}_{Y}^{2}\right\rangle\right)$ is the sum of the variances $\left.\sigma_{Z}^{2}\right|_{L 1}$ $\left(\left.\sigma_{Y}^{2}\right|_{L 1}\right)$ and $\left.\sigma_{Z}^{2}\right|_{L 2,3}\left(\left.\sigma_{Y}^{2}\right|_{L 2,3}\right)$, where the angle brackets represent ensemble averaging, $\left.\sigma_{Z}^{2}\right|_{L 1}=\left\langle\left[Z^{L 1}-\left\langle Z^{L 1}\right\rangle\right]^{2}\right\rangle \quad\left(\left.\sigma_{Y}^{2}\right|_{L 1}=\right.$ $\left.\left\langle\left[Y^{L 1}-\left\langle Y^{L 1}\right\rangle\right]^{2}\right\rangle\right)$ and $\left.\sigma_{Z}^{2}\right|_{L 2,3}=\left\langle\left[Z^{L 2,3}-\left\langle Z^{L 2,3}\right\rangle\right]^{2}\right\rangle \quad\left(\left.\sigma_{Y}^{2}\right|_{L 2,3}=\right.$ $\left.\left\langle\left[Y^{L 2,3}-\left\langle Y^{L 2,3}\right\rangle\right]^{2}\right\rangle\right)$. If we additionally assume that the errors of the vortex position estimation from the pulsed lidar data and from the triangulation are equal, then we have $\left.\sigma_{Z}\right|_{L 1}=$ $\left.\sigma_{Z}\right|_{L 2,3} \approx \hat{\sigma}_{Z} / \sqrt{ } 2 \approx 6 \mathrm{~m}$ and $\left.\sigma_{Y}\right|_{L 1}=\left.\sigma_{Y}\right|_{L 2,3} \approx \hat{\sigma}_{Y} / \sqrt{ } 2 \approx 9 \mathrm{~m}$. Such errors of the core position measurement $(6 \mathrm{~m}$ for vertical and $9 \mathrm{~m}$ for horizontal coordinates) with one pulsed lidar or two cw lidars are likely to be overestimated for two reasons: 1) timing error and 2) measurement with lidar L1 and with lidars L2 and L3 in different vertical planes (Fig. 1). Because the time synchronization between the lidars was not ideal, a timing error of approximately $1 \mathrm{~s}$ was possible. This could result, for example, for a vertical descent speed of $1 \mathrm{~m} / \mathrm{s}$ and a horizontal crosswind component of $4 \mathrm{~m} / \mathrm{s}$ in a vortex shift of $1 \mathrm{~m}$ along the vertical axis and $4 \mathrm{~m}$ in horizontal direction. Because of practical restrictions, it was not possible to place the pulsed lidar in the plane of the cw lidars scans. Although the angle between the planes is relatively small $(7.7 \mathrm{deg})$, the plane separation is approximately $27 \mathrm{~m}$, for example, at a lateral distance of $200 \mathrm{~m}$ from the lidar L3 (Fig. 1). The difference of vortex core $Y$ coordinates measured in different planes (separated along the $X$ axis) can be a few meters. For example, due to turbulence effects the crosswind velocities in two separated planes may differ by $0.1 \mathrm{~m} / \mathrm{s}$. (This is a reasonable value for the separation of $27 \mathrm{~m}$.) Then, after a period of $30 \mathrm{~s}$, the vortex core $Y$ coordinates can differ by $(0.1 \mathrm{~m} / \mathrm{s} \times 30 \mathrm{~s})=3 \mathrm{~m}$. When equivalent contributions are assumed from each of the four factors of uncertainty, that is, accuracy of both lidar types, timing, and geometrical factor, to $\sigma_{Z}^{2}$ and $\sigma_{Y}^{2}$, the error of vortex position measurements by the pulsed lidar and the $\mathrm{cw}$ lidars becomes approximately $4.5 \mathrm{~m}$ in the vertical and $6.5 \mathrm{~m}$ in the 
horizontal. These errors are close to the accuracy estimate $( \pm 4 \mathrm{~m})$ obtained in Ref. 19 for the vortex core position measured by cw lidars and using the triangulation method. The assumption about the independence of the random errors of the estimates $\left\{Z^{L 1}\left(t_{k}\right), Y^{L 1}\left(t_{k}\right)\right\}$ and $\left\{Z^{L 2,3}\left(t_{k}\right), Y^{L 2,3}\left(t_{k}\right)\right\}$ is correct. However, from our measured data, it is not possible to find exactly the contribution of the pulsed and cw lidars to the discrepancies $\hat{\sigma}_{Z} \approx 9 \mathrm{~m}$ and $\hat{\sigma}_{Y} \approx 13 \mathrm{~m}$. Probably, the estimates of the core position measurement accuracy $(4.5 \mathrm{~m}$ and $6.5 \mathrm{~m}$ ) are too rough.

The vortex circulation estimate $\hat{\Gamma}$ is determined as

$$
\hat{\Gamma} \sim \sum_{i}\left|V_{ \pm}^{L m}\left(\varphi_{i}\right)\right| \times R_{C l}^{L m}\left|\sin \left(\varphi_{i}-\varphi_{C l}^{L m}\right)\right|
$$

Therefore, the accuracy of circulation measurement depends on the accuracy of estimation of the parameters: $V_{ \pm}^{L m}(\varphi), \varphi_{C l}^{L m}$, and $R_{C l}^{L m}$. Even if the data for the velocity envelope $V_{ \pm}^{ \pm m}(\varphi)$ are poor, in many cases the angle $\delta \varphi_{i}=\varphi_{i}-\varphi_{C l}^{L m}, m=1,2,3$, can be estimated with high accuracy $( \pm 0.04 \mathrm{deg}$ in the case of pulsed lidar and $\pm 0.15 \mathrm{deg}$ in the case of cw lidar), and the effect of the error of this angle estimation on the circulation estimate $\hat{\Gamma}$ can be neglected. The distance $R_{C l}^{L 1}$ is estimated from data of the pulsed lidar, and $R_{C l}^{L 2}$ and $R_{C l}^{L 3}$ are the triangulation results obtained from the QinetiQ and ONERA $\mathrm{cw}$ lidar data. Because of the measurement geometry, the errors of $R_{C l}^{L 1}$ and $Y^{L 1}$ estimation are almost the same. Let the contribution of the pulsed lidar to the discrepancy $\hat{\sigma}_{Y} \approx 13 \mathrm{~m}$ be larger than the contribution of the cw lidars and the error of $R_{C l}^{L 1}$ (or $Y^{L 1}$ ) be equal to $10 \mathrm{~m}$. Then at $R_{C l}^{L 1} \sim 1 \mathrm{~km}$, the relative measurement error of the distance between the pulsed lidar and the vortex core is $\sim 1 \%$. In accordance with the theory, ${ }^{19}$ the errors of estimation of $R_{C l}^{L 2}$ and $R_{C l}^{L 3}$ is $\sim 4 \mathrm{~m}$. In our experiments $R_{C l}^{L 3} \in(100 \mathrm{~m}, 350 \mathrm{~m})$ with a mean value of $R_{C l}^{L 3} \sim 200 \mathrm{~m}$. Therefore, the relative error of $R_{C l}^{L 3}$ estimation is $\sim 2 \%$. Thus, the effect of the error of $R_{C l}^{L 3}$ estimation $(m=1,2,3)$ on the circulation estimation accuracy is negligible. Even if the absolute error of estimation of the vortex core coordinates $\left\{Z^{L 1}\left(t_{k}\right), Y^{L 1}\left(t_{k}\right)\right\}$ or $\left\{Z^{L 2,3}\left(t_{k}\right), Y^{L 2,3}\left(t_{k}\right)\right\}$ is large, for example, because of some technical problems in the scanner or timing device, it has almost no influence on the circulation estimation accuracy because the relative errors of measurement of the angle $\delta \varphi_{i}$ and $R_{C l}^{L m}$ are very small.

The main error source of the circulation estimation is the error in the estimation of the velocity envelope $V_{ \pm}^{L m}(\varphi)$. Because of the high SNR and the condition $\delta R_{C l}^{L 1} \ll \Delta R$ where $\delta R_{C l}^{L 1}$ is the error of estimation of $R_{C l}^{L 1}$ and $\Delta R=88 \mathrm{~m}$ is the sensing volume size in the case of pulsed lidar, the envelope $V_{ \pm}^{L 1}(\varphi)$ was measured with relatively high accuracy. Also good accuracy for $V_{ \pm}^{L 3}(\varphi)$ was achieved due to the corresponding change of the focal length $R$ during the measurements with the ONERA lidar. Thus, we can expect accurate measurements of the vortex circulation with both the DLR pulsed lidar and the ONERA cw lidar.

\section{Comparison of Vortex Circulation}

The circulation is the most important parameter for wake-vortex characterization because it describes the vortex strength and, therefore, is an index characterizing the degree of potential hazard to following aircraft. The evolution, decay, and lifetime of wake vortices for different aircraft configurations and atmospheric conditions are topics of considerable current interest. ${ }^{26-28}$ The use of CDLs for the experimental investigation of vortex circulation under real atmospheric conditions gives an unique possibility to review conflicting theories about vortex evolution and decay, namely, the different concepts frequently termed predictable decay and stochastic collapse. ${ }^{26,27}$ The significance of the results and conclusions of such investigations essentially depends on the lidar accuracy. Therefore, the accuracy of the lidar measurements of vortex circulation will be estimated by comparison of the measurement results of the pulsed and cw lidars.

In the following discussion, the vortex circulation and its temporal behavior are presented in relative units by normalization with the theoretical root (initial) circulation $\Gamma_{0}$ (square meters per second) and the reference timescale $t_{0}$ (seconds). The latter describes the time in which the vortex pair descends at the distance of one initial vortex core separation. The parameters $\Gamma_{0}$ and $t_{0}$ are defined as ${ }^{27}$

$$
\begin{gathered}
t_{0}=4 \pi s^{3}\left(B^{3} / C_{L} V A\right) \\
\Gamma_{0}=M g / s \rho B V
\end{gathered}
$$

where $s$ is the spanwise load factor, $B$ is the wing span (meters) $C_{L}$ is the lift coefficient, $V$ is the aircraft speed (meters per second), $A$ is the wing area (square meters) $M$ is the aircraft mass (kilogram), $g$ is the gravitational acceleration (meters per second squared), and $\rho$ is the air density (kilogram per cubic meter). In this way, it is possible to make the results shown in Figs. 6-9 independent of a particular aircraft type.

Figure 6 shows three examples of normalized vortex circulation measured with the DLR pulsed lidar L1 (squares) and the ONERA cw lidar L3 (circles). These measurements were carried out on 13 June 2002 (Fig. 6a), 14 June 2002 (Fig. 6b), and 17 June 2002. Each point (both squares and circles) in Fig. 6 represents a mean value between circulation estimates of the right and left vortices. Because of the different measurement geometry, the pulsed lidar can measure the vortex circulation during longer time periods compared with the cw lidar (Figs. 6a and 6b). In the case shown in Fig. 6c, the crosswind was rather weak and the wake vortices were within the measurement area of both lidars for a long time period almost until the complete vortex decay. The long duration of the circulation measurements, their high accuracy, and the relatively weak disturbances caused by ambient turbulence allow the clear identification of two phases of the vortex evolution: the moderate decrease (until 4-5 time units in the examples shown in Fig. 6) that turns into a steeper slope characterizing the phase of rapid decay. These
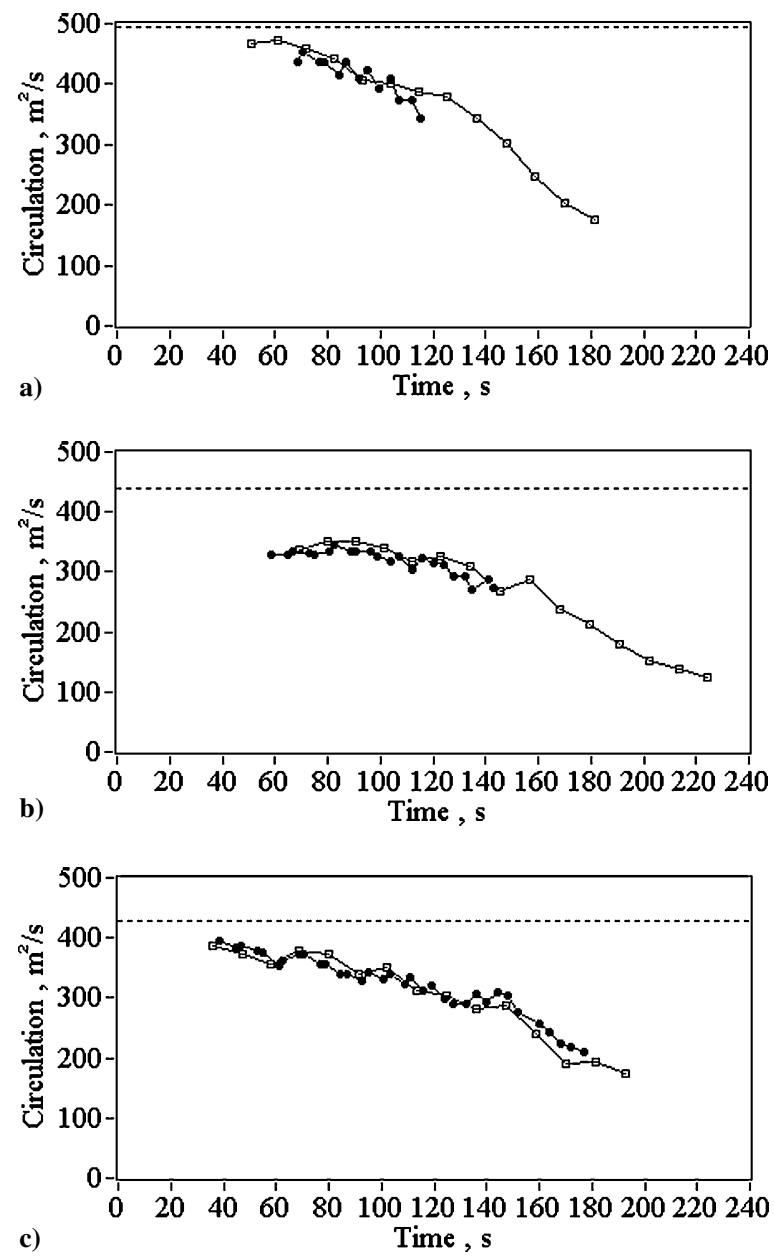

Fig. 6 Examples of normalized wake-vortex circulation measured by $\square$, DLR pulsed lidar and $\bigcirc$, ONERA cw lidar: a) 13 June 2002, b) 14 June 2002, and c) 17 June 2002. 

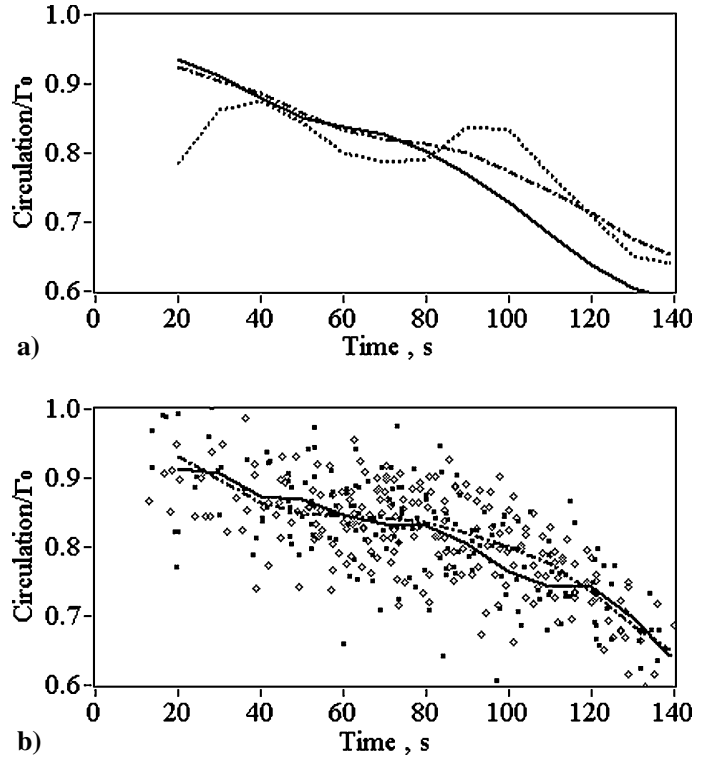

Fig. 7 Normalized mean circulation measured by $\longrightarrow$, DLR; - ·-•, ONERA; and ---, QinetiQ lidars and single normalized circulation estimates (squares and rhombuses) vs normalized time after over-flight (vortex age): a) all measured data and b) only data measured by ONERA (rhombuses) and DLR (squares) lidars for the same time intervals used for averaging.

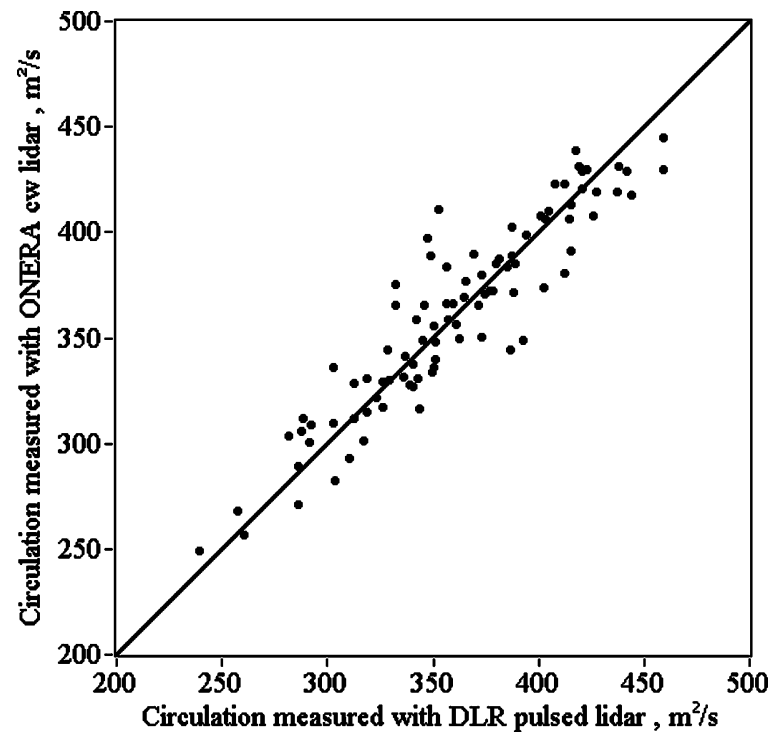

Fig. 8 Comparison of simultaneous single circulation estimates obtained from data of ONERA and DLR lidar measurements.

measurements clearly corroborate the circulation evolutions found in numerical simulations ${ }^{28-30}$ and give an indication for the validity of the concept of the two-phase decay.

To compare the mean normalized circulation measured with different lidars, we have averaged all normalized circulation estimates falling into the time intervals $\left[t_{s}+k \Delta t, t_{s}+(k+1) \Delta t\right]$ (where $t_{s}=15 \mathrm{~s}, \Delta t=10 \mathrm{~s}$, and $\left.k=0,1,2, \ldots, 12\right)$ and plotted the results. Figure 7 a shows the normalized mean circulation $\Gamma / \Gamma_{0}$ vs normalized time after overflight (or vortex age). The scatter of single circulation estimates obtained from the data of the pulsed lidar and the ONERA cw lidar is similar (for example, Fig. 6) and less than the scatter in the case of circulation measurement with QinetiQ cw lidar. The QinetiQ lidar was positioned to optimize vortex positional information for triangulation purposes at the expense of accuracy of circulation observations. For vortex ages up to almost 3 time units, there is very good agreement between the DLR pulsed lidar and the ONERA cw lidar results. (Compare the solid and dot-dashed

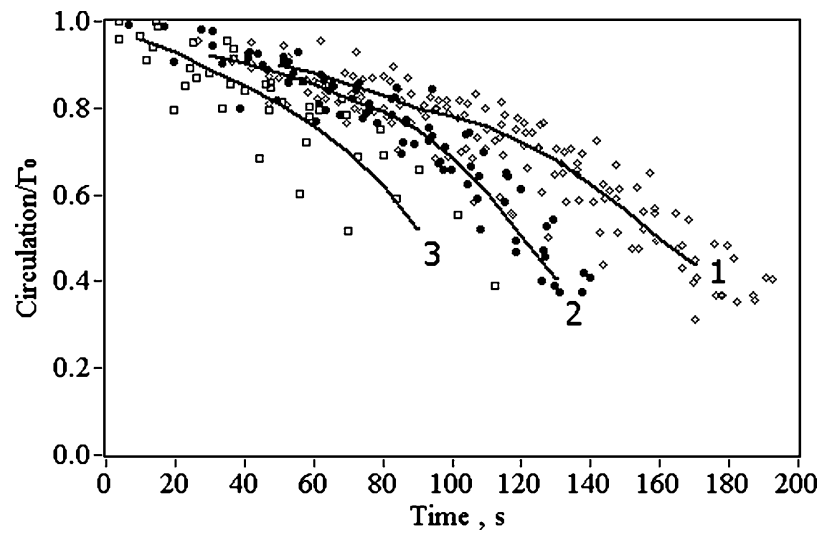

Fig. 9 Influence of atmospheric turbulence on behavior of normalized wake-vortex circulation at 1) $\left.\varepsilon=0.5-2 \times 10^{-4} \mathrm{~m}^{2} / \mathrm{s}^{3}, 2\right) \varepsilon=2-$ $5 \times 10^{-4} \mathrm{~m}^{2} / \mathrm{s}^{3}$ and 3) $\varepsilon=5-20 \times 10^{-4} \mathrm{~m}^{2} / \mathrm{s}^{3}$.

curves in Fig. 7a). However, with increasing time the deviation between these curves becomes larger. This arises because, after 3 time units, many circulation estimates (with rapid decay) are available for averaging of the pulsed lidar data, a significant fraction of which could not be obtained from cw lidar data because of the measurement geometry. Figure $7 \mathrm{~b}$ shows that there is good agreement of the results when only data measured in the same time intervals are used for averaging of the circulation estimates.

Figure $7 \mathrm{~b}$ shows in addition to the mean normalized circulation (solid and dot-dashed curves) the single circulation estimates that were used to obtain these curves. Because the initial circulation $\Gamma_{0}$ was different for various overflights $\left(350-500 \mathrm{~m}^{2} / \mathrm{s}\right)$, we used normalized circulation estimates for calculating the scatter in the measurement results. The standard deviations of the single normalized circulation estimates from the mean curves in Fig. $7 \mathrm{~b}$ are 0.063 and 0.057 in the case of measurements with the DLR pulsed lidar and the ONERA cw lidar, respectively. This means that the scatter is almost the same for both lidars and the standard deviation of the normalized circulation estimates is around 0.06 . From the mean value of all circulation estimates of $360 \mathrm{~m}^{2} / \mathrm{s}$, we can roughly estimate a standard deviation (assuming that all overflights would be at the same initial vortex circulation $\Gamma_{0}$ ) in the case of measurement with one of the lidars by multiplying 0.06 by $360 \mathrm{~m}^{2} / \mathrm{s}$. This standard deviation is approximately $22 \mathrm{~m}^{2} / \mathrm{s}$ and apparently has to depend mainly on the lidar measurement accuracy, the atmospheric turbulence, and the variability of vortex evolution along the flight direction. ${ }^{20}$ For the analysis of the circulation measurement accuracy, the effect of timing error and geometrical factor can be neglected.

The error for circulation estimation can be found by comparison of the simultaneous single estimates obtained from the data of the DLR pulsed lidar and the ONERA cw lidar measurements. Figure 8 shows the result of this comparison. By analogy with Eqs. (1) and (2), let us introduce the parameter $\hat{\sigma}_{\Gamma}$ characterizing the level of discrepancy in the vortex circulation measurements as

$$
\hat{\sigma}_{\Gamma}=\sqrt{\frac{1}{K} \sum_{k=1}^{K}\left[\Gamma^{L 1}\left(t_{k}\right)-\Gamma^{L 3}\left(t_{k}\right)\right]^{2}}
$$

where $\Gamma^{L 1}\left(t_{k}\right)$ and $\Gamma^{L 3}\left(t_{k}\right)$ are circulation estimates (circles in Fig. 8) from lidar L1 and lidar L3 data, respectively, and $K$ is a number of estimates used for the averaging. In accordance with the data of Fig. 8, $K=95$. The calculation by Eq. (5) gives the result: $\hat{\sigma}_{\Gamma} \approx 18 \mathrm{~m}^{2} / \mathrm{s}$. From data of Fig. 7b, we have obtained almost equal standard deviation of normalized circulation $(\sim 0.06)$ estimated from the data measured with the DLR pulsed lidar and the ONERA cw lidar. When it is into account that the measurements with two lidars are simultaneous (at the same atmospheric condition), one can conclude that the errors of circulation estimation from data of the DLR and ONERA lidars are the same. The random errors of the circulation measurement with both lidars are independent. Then, the error of 
the circulation measurement with the DLR pulsed or the ONERA cw lidar can be estimated as $\hat{\sigma}_{\Gamma} / \sqrt{ } 2=\left(18 \mathrm{~m}^{2} / \mathrm{s}\right) / \sqrt{ } 2 \approx 13 \mathrm{~m}^{2} / \mathrm{s}$.

Because the experiments were carried out under different atmospheric conditions, the scatter of the circulation estimates depends on both the lidar measurement accuracy and the wind turbulence. The vortex circulation measurement error of $13 \mathrm{~m}^{2} / \mathrm{s}$ is 1.7 times smaller than the scatter of $22 \mathrm{~m}^{2} / \mathrm{s}$. Therefore, the measurement accuracy is sufficient for the analysis of circulation measurements under different levels of wind turbulence.

\section{Effect of Turbulence on Vortex Circulation}

In the atmospheric boundary layer, the main source of turbulence is the frictional forces created by the Earth's surface on the air transported by the wind. ${ }^{31,32}$ The temperature stratification can either increase or decrease the turbulence strength. The range of scales of turbulent wind inhomogeneities is wide: from a few centimeters to an order of $100 \mathrm{~m}$ (Ref. 33). The largest turbulent eddies transfer the wake vortices almost without an effect on the vortex structure. The vortex structure can be distorted and finally completely destroyed by eddies with scales of the inertial interval of the turbulence, where the turbulence level can be described by one parameter, the turbulent energy dissipation rate $\varepsilon$.

The turbulent energy dissipation rate was estimated from data measured with the DLR pulsed Doppler lidar, using the method described in Ref. 8. At first, the spatial structure functions $D_{V}\left(R_{i}\right)=\left\langle\left[V_{r}\left(R_{i}+R\right)-V_{r}(R)\right]^{2}\right\rangle$, where $i=1,2$, are obtained from the measured radial wind velocity $V_{r}(R)$. Then the turbulent energy dissipation rate can be calculated by the formula

$$
\varepsilon=\left[\frac{D_{V}\left(R_{2}\right)-D_{V}\left(R_{1}\right)}{C_{K}\left(R_{2}^{\frac{2}{3}}-R_{1}^{\frac{2}{3}}\right)}\right]^{\frac{3}{2}}
$$

where $C_{K} \approx 2$ is the Kolmogorov constant, $R_{1}=60 \mathrm{~m}$, and $R_{2}=120 \mathrm{~m}$.

For each overflight, the height profile of the turbulent energy dissipation rate $\varepsilon(h)$ is retrieved (where $h$ is height and $40<h<400 \mathrm{~m}$ ) and then $\varepsilon(h)$ is averaged over the heights of wake-vortex observations out of ground effect. The obtained values are within the range of $0.5 \times 10^{-4}-20 \times 10^{-4} \mathrm{~m}^{2} / \mathrm{s}^{3}$. This range is split in three parts: $0.5 \times 10^{-4}-2 \times 10^{-4} \mathrm{~m}^{2} / \mathrm{s}^{3}, 2 \times 10^{-4}-5 \times 10^{-4} \mathrm{~m}^{2} / \mathrm{s}^{3}$, and $5 \times 10^{-4}-20 \times 10^{-4} \mathrm{~m}^{2} / \mathrm{s}^{3}$, and the circulation estimates obtained from the pulsed lidar data are sorted in three groups in accordance with such splitting. For each level of turbulence strength, the mean circulation vs time is calculated. Because the pulsed lidar can measure the wake vortices over longer time periods than the cw lidars, here the analysis of turbulence effects on the vortex circulation is restricted only to pulsed lidar data.

Figure 9 shows the normalized vortex circulation vs time for the different atmospheric turbulence conditions that occurred during the experiment. The rhombuses, circles, and squares represent the single normalized estimates, and curves 1-3 are the mean curves of the normalized circulation. It can be seen that the vortex decay is faster at stronger turbulence levels. At the turbulent energy dissipation rate of $(5-20) \times 10^{-4} \mathrm{~m}^{2} / \mathrm{s}^{3},(2-5) \times 10^{-4} \mathrm{~m}^{2} / \mathrm{s}^{3}$, and $(0.5-2) \times 10^{-4} \mathrm{~m}^{2} / \mathrm{s}^{3}$, the normalized vortex circulation reaches the 0.5 level at approximately $2.5,3.5$, and 4.5 time units, respectively. Because each curve represents the mean of approximately 10 single measurements, no pronounced slope break can be observed in these mean curves. Nevertheless, the two-phase decay of the underlying individual cases becomes evident from the curvature of the mean circulation evolutions.

\section{Conclusions}

The results of the wake-vortex measurements with the $2-\mu \mathrm{m}$ pulsed Doppler lidar from DLR and the $10-\mu \mathrm{m}$ cw Doppler lidars from ONERA and QinetiQ have been compared. The standard deviation of the difference between the core position estimates obtained from data measured with the pulsed and cw lidars is $9 \mathrm{~m}$ in the vertical and $13 \mathrm{~m}$ in the horizontal direction. The errors of the circulation measurements with the DLR and ONERA lidars are almost the same and equal $13 \mathrm{~m}^{2} / \mathrm{s}$. This accuracy and the long observation times allow us to analyze the temporal evolution of vortex circulation, in general and under different atmospheric turbulence conditions. The wake-vortex measurements were carried out under moderate $\left[\varepsilon=(5-20) \times 10^{-4} \mathrm{~m}^{2} / \mathrm{s}^{3}\right]$, weak $\left[\varepsilon=(2-5) \times 10^{-4} \mathrm{~m}^{2} / \mathrm{s}^{3}\right]$, and very weak $\left[\varepsilon=(0.5-2) \times 10^{-4} \mathrm{~m}^{2} / \mathrm{s}^{3}\right]$ turbulence conditions. The mean normalized vortex circulation decreases with time after overflight and reaches a 0.5 level approximately 1.8 times faster at moderate turbulence than at very weak turbulence. In all turbulence regimes, we clearly identify two phases of circulation decay: an initial phase of moderate decay that is followed by a phase of rapid decay. Herewith, lidar measurements for the first time clearly corroborate the characteristics of circulation evolutions previously found in numerical simulations. ${ }^{29,30}$

The main conclusion of this comparison work is that the results of wake-vortex measurements with the pulsed and cw lidars are in good agreement. Improvements of the lidar systems, the measurement methods, and the data processing algorithms, as well as operating under favorable atmospheric conditions (in particular, a lot of aerosol particles) allowed the measurement of the wake-vortex properties with high accuracy.

\section{Acknowledgments}

The simultaneous measurements of aircraft wake vortices by pulsed and cw lidars have been carried out in the framework of the C-Wake Project (Contract G4RD-CT-1999-00141) within the Fifth Research Framework Programme of the European Union. QinetiQ participation was also sponsored by the CARAD program of the United Kingdom's Department of Trade and Industry.

\section{References}

${ }^{1}$ Köpp, F., Schwiesow, R. L., and Werner, C., "Remote Measurements of Boundary Layer Wind Profiles Using a CW Doppler Lidar," Journal of Climate Applied Meteorology, Vol. 23, No. 1, 1984, pp. 148-158.

${ }^{2}$ Hall, F. F., Huffaker, R. M., Hardesty, R. M., Jackson, M. E., Lawrence, T. R., Post, M. J., Richter, R. A., and Weber, B. F., "Wind Measurement Accuracy of the NOAA Pulsed Infrared Doppler Lidar," Applied Optics, Vol. 23, No. 15, 1984, pp. 2503-2506.

${ }^{3}$ Hawley, J. G., Tang, R., Henderson, S. W., Hale, C. P., Kavaya, M. J., and Moerder, D., "Coherent Launch-Site Atmospheric Wind Sounder: Theory and Experiment," Applied Optics, Vol. 32, No. 24, 1993, pp. 4557-4568.

${ }^{4}$ Frehlich, R., Hannon, S. M., and Henderson, S. W., "Performance of a $2-\mu \mathrm{m}$ Coherent Doppler Lidar for Wind Measurements," Journal of Atmospheric and Oceanic Technology, Vol. 11, Dec. 1994, pp. 1517-1528.

${ }^{5}$ Werner, C., Flamant, P. H., Reitebuch , Köpp, F., Streicher, J., Rahm, S., Nagel, E., Klier, M., Herrmann, H., Loth, C., Delville, P., Drobinski, P., Romand, B., Boitel, C., Oh, D., Lopez, M., Meissonner, M., Bruneau, D., and Dabas, A., "Wind Infrared Doppler Lidar Instrument," Optical Engineering, Vol. 40, No. 1, 2001, pp. 115-125.

${ }^{6}$ Smalikho, I., "Techniques of Wind Vector Estimation from Data Measured with a Scanning Coherent Doppler Lidar," Journal of Atmospheric and Oceanic Technology, Vol. 20, Feb. 2003, pp. 276-291.

${ }^{7}$ Gal-Chen, T., Xu, M., and Eberhard, W. L., "Estimations of Atmospheric Boundary Layer Fluxes and Other Turbulence Parameters From Doppler Lidar Data," Journal of Geophysical Research, Vol. 97, No. D17, 1992, pp. $18,409-18,423$.

${ }^{8}$ Banakh, V. A., and Smalikho, I. N., "Estimation of the Turbulence Energy Dissipation Rate from the Pulsed Doppler Lidar Data," Atmospheric and Oceanic Optics, Vol. 10, No. 12, 1997, pp. 957-965.

${ }^{9}$ Banakh, V. A., Smalikho, I. N., Köpp, F., and Werner, C., "Measurements of Turbulent Energy Dissipation Rate with a CW Doppler Lidar in the Atmospheric Boundary Layer," Journal of Atmospheric and Oceanic Technology, Vol. 16, Aug. 1999, pp. 1044-1061.

${ }^{10}$ Frehlich, R., and Cornman, L., "Estimating Spatial Velocity Statistics with Coherent Doppler Lidar," Journal of Atmospheric and Oceanic Technology, Vol. 19, No. 3, 2002, pp. 355-366.

${ }^{11}$ Hannon, S. M., and Thomson, J. A., "Aircraft Wake Vortex Detection and Measurement with Pulsed Solid-State Coherent Laser Radar," Journal of Modern Optics, Vol. 41, No. 11, 1994, pp. 2175-2196.

${ }^{12}$ Köpp, F., "Doppler Lidar Investigation of Wake Vortex Transport Between Closely Spaced Runways," AIAA Journal, Vol. 32, No. 4, 1994, pp. 805-810.

${ }^{13}$ Constant, G., Foord, R., Forrester, P. A., and Vaughan, J. M., "Coherent Laser Radar and the Problem of Aircraft Wake Vortices," Journal of Modern Optics, Vol. 41, No. 11, 1994, pp. 2153-2173. 
${ }^{14}$ Köpp, F., "Wake-Vortex Characteristics of Military-Type Aircraft Measured at Airport Oberpfaffenhofen Using the DLR Laser Doppler Anemometer," Aerospace Science and Technology, Vol. 3, 1999, pp. 191-199.

${ }^{15}$ Harris, M., Vaughan, J. M., Huenecke, K., and Huenecke, C., "Aircraft Wake Vortices: a Comparison of Wind-Tunnel Data with Field-Trial Measurements by Laser Radar," Aerospace Science and Technology, Vol. 4, 2000, pp. 363-370.

${ }^{16}$ Vaughan, J. M., and Harris, M., "Lidar Measurement of B747 Wakes: Observation of a Vortex Within a Vortex," Aerospace Science and Technology, Vol. 5, 2001, pp. 409-411.

${ }^{17}$ Harris, M., Young, R. I., Köpp, F., Dolfi, A., and Cariou, J.-P., "Wake Vortex Detection and Monitoring," Aerospace Science and Technology, Vol. 6, 2002, pp. 325-331.

${ }^{18}$ Keane, M., Buckton, D., Redfern, M., Bollig, C., Wedekind, C., Köpp, F., and Berni, F., "Axial Detection of Aircraft Wake Vortices Using Doppler Lidar," Journal of Aircraft, Vol. 39, No. 5, 2002, pp. 850-861.

${ }^{19}$ Köpp, F., Smalikho, I., Rahm, S., Dolfi, A., Cariou, J.-P., Harris, M., Young, R. I., Weekes, K., and Gordon, N., "Characterization of Aircraft Wake Vortices by Multiple-Lidar Triangulation," AIAA Journal, Vol. 41, No. 2, 2003, pp. 1081-1088.

${ }^{20}$ Holzäpfel, F., Gerz, T., Köpp, F., Stumpf, E., Harris, M., Young, R. I., and Dolfi, A., "Strategies for Circulation Evaluation of Aircraft Wake Vortices Measured by Lidar," Journal of Atmospheric and Oceanic Technology, Vol. 20, 2003, pp. 1183-1195.

${ }^{21}$ Köpp, F., Rahm, S., and Smalikho, I., "Characterisation of Aircraft Wake Vortices by $2-\mu \mathrm{m}$ Pulsed Doppler Lidar," Journal of Atmospheric and Oceanic Technology, Vol. 21, 2004, pp. 194-206.

${ }^{22}$ Henderson, S. W., Suni, P. J. M., Hale, C. P., Hannon, S. M., Magee, J. R., Bruns, D. L., and Yuen, E. H., "Coherent Laser Radar at $2 \mu \mathrm{m}$ Using
Solid-State Lasers," IEEE Transactions on Geoscience and Remote Sensing, Vol. 31, No. 1, 1993, pp. 4-15.

${ }^{23}$ Sonnenschein, C. M., and Horrigan, F. A., "Signal-to-Noise Relationships for Coaxial Systems that Heterodyne Backscatter from the Atmosphere," Applied Optics, Vol. 10, No. 7, 1971, pp. 1600-1604.

${ }^{24}$ Gerz, T., "Wake Vortex Prediction and Operation: Towards an Operational System," Proceedings of the 3rd ONERA-DLR Aerospace Symposium, ONERA, Paris, June 2001, Paper S1-3.

${ }^{25}$ Jazwinski, A. H., Stochastic Processes and Filtering Theory, Academic Press, New York, 1970, pp. 272-279.

${ }^{26}$ Spalart, P. R., "Airplane Trailing Vortices," Annual Review of Fluid Mechanics, Vol. 30, 1998, pp. 107-138.

${ }^{27}$ Gerz, T., Holzäpfel, F., and Darracq, D., "Commercial Aircraft Wake Vortices," Progress in Aerospace Sciences, Vol. 38, 2002, pp. 181-208.

${ }^{28}$ Holzäpfel, F., Hofbauer, T., Darracq, D., Moet, H., Garnier, F., and Ferreira Gago, C., "Analysis of Wake Vortex Decay Mechanisms in the Atmosphere," Aerospace Science and Technology, Vol. 7, 2003, pp. 263-275.

${ }^{29}$ Proctor, F. H., and Switzer, G. F., "Numerical Simulation of Aircraft Trailing Vortices," Proceedings of the 9th Conference on Aviation, Range and Aerospace Meteorology, AMS, Boston, Sept. 2000, Paper 7.12.

${ }^{30}$ Holzäpfel, F., Gerz, T., and Baumann, R., "The Turbulent Decay of Trailing Vortex Pairs in Stably Stratified Environments," Aerospace Science and Technology, Vol. 5, 2001, pp. 95-108.

${ }^{31}$ Lumley, J. L., and Panofsky, H. A., The Structure of Atmospheric Turbulence, Willey-Interscience, New York, 1964, pp. 59-212.

${ }^{32}$ Monin, A. S., and Yaglom, A. M., Statistical Fluid Mechanics, Mechanics of Turbulence, Vol. 2, MIT Press, Cambridge, MA, 1971, pp. 257-412.

${ }^{33}$ Byzova, N. L., Ivanov, V. N., and Garger, E. K., Turbulence in the Boundary Layer of the Atmosphere, Gidrometeoizdat, Leningrad, 1989, pp. 28-134. 